\title{
Research on the drags of high speed elevator with different height diversion cover
}

\author{
Zhangwei Ling ${ }^{1,}$, Ping Tang ${ }^{1,}$, Xuebin Wang ${ }^{1}$, Zheng Lin ${ }^{1}$, Di Tang ${ }^{2}$ \\ ${ }^{1}$ Zhejiang Provincial Special Equipment Inspection and Research Institute, Kai xuan Road 211, \\ Hangzhou, 310020, P. R. China \\ ${ }^{2}$ Zhejiang University of Technology, College of Mechanical Engineering, Hangzhou, \\ Zhejiang 310032, P. R. China \\ alingzhangwei@163.com
}

Keywords: High speed evaluator, Aerodynamic performance, Diversion cover; CFD

\begin{abstract}
The three-dimensional flow of high speed elevator is numerical simulated using the Computational Fluid Dynamics Method (CFD). The car of high speed elevator has dimensions of length $2.3 \mathrm{~m}$, width $1.7 \mathrm{~m}$ and height $2.4 \mathrm{~m}$. The shape of diversion cover is conic. The flows of high speed elevator with different height diversion cover are simulated, and then the drags are analyzed. It is present that the drags of the car are decreased when the height of diversion cover increases.
\end{abstract}

\section{Introduction}

A large amount of high buildings are built with the rapid development of China economy, the height of high buildings is generally achieved $500 \mathrm{~m}$. One of the highest buildings is ShangHai Tower[1], the building stands $632 \mathrm{~m}$ high and has 123 stories. Thus the high speed elevator systems (over $2 \mathrm{~m} / \mathrm{s}$ ) have been predominantly required in China. However, with the increase of the elevator speed, the problems of high-speed stability, security, and noise need to be considered. The aerodynamic performance of high speed elevator plays an important role, and the height of the diversion cover is the key factor. Thus it is necessary to study the influences of the cover height on the elevator's performance.

The experimental method [2] and numerical method [3,4] have been performed in the aerodynamic performances analysis and the car geometry optimization. However the costs of the experiments are much higher, the Fluent and the CFX Software have been widely used in the numerical simulation with the numerical method been matured [5]. The turbulent flows and aerodynamic performances have been studied using the CFD method. The forces and fluid-structure coupled vibrations have been studied by W.D.Zhu[6], it shows that the turbulent flow is the source of vibration. The traditional Euler equations and N-S equations have been used in the complex flow simulation of high speed elevator by So.A.T.P[7]. The diversion cover with conic surface, semi-spherical surface, and parabolic surface are further considered. The results show that the aerodynamic performance of diversion covers with conic surface is better. However the influence of diversion cover height have not been studied yet.

In this paper the conic surface diversion cover of different heights are studied. then turbulent flows of the high speed elevators are simulated with Fluent. And the drags of the elevator car are studied.

\section{The evaluator model}

The car of the high speed elevator studied in this paper is a box with the length of $2.3 \mathrm{~m}$, with the width of $1.7 \mathrm{~m}$, and height of $2.4 \mathrm{~m}$. The diversion covers are built using conic surfaces with the height of $0.2 \mathrm{~m}, 0.4 \mathrm{~m}, 0.6 \mathrm{~m}, 0.8 \mathrm{~m}, 1.0 \mathrm{~m}$, and $1.2 \mathrm{~m}$, respectively. To reduce the computational cost, only quarters of the models are built, parts of them are shown in Fig 1. 


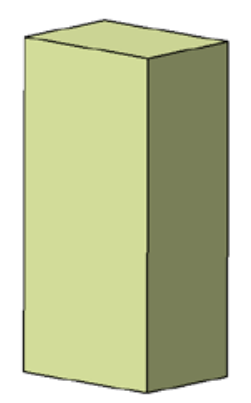

a) Without cover

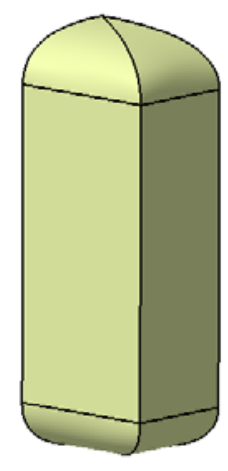

b) $h=0.4 m$

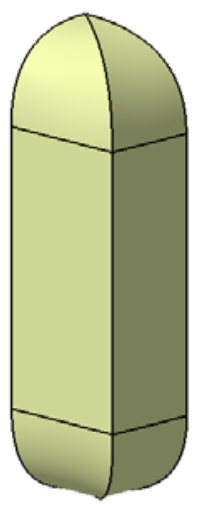

c) $h=0.8 m$

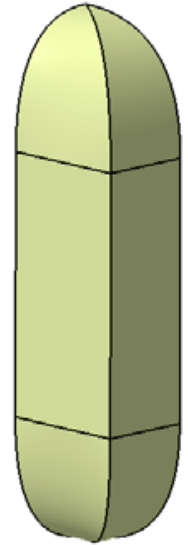

d) $h=1.2 \mathrm{~m}$

Fig.1. High speed evaluator models with different diversion cover

The elevator well has dimensions of length $3 \mathrm{~m}$, width $2.6 \mathrm{~m}$, and height $100 \mathrm{~m}$, as shown in Fig 2(a). The meshes are generated with structure mesh with about 267,000 cells. The meshs of diversion cover with height of 1.2m are shown in Fig 2(b), 2(c).



a) Flow region

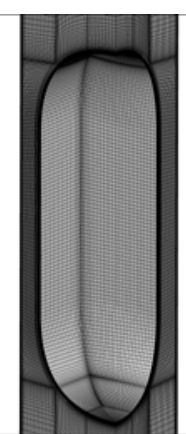

b) mesh of the elevator car Fig.2. model of elevator with $\mathrm{h}=1.2 \mathrm{~m}$

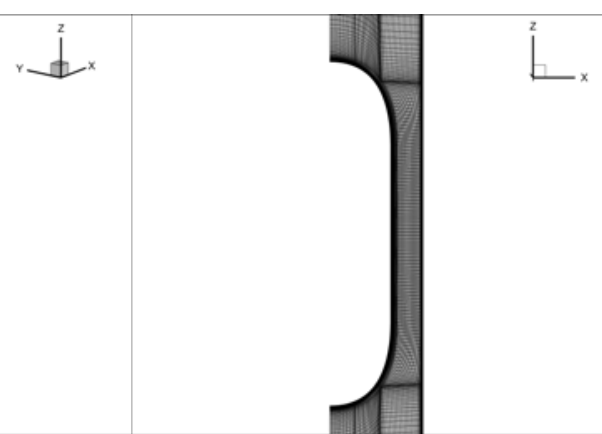

c) mesh of the symmetry plane

\section{Computation method}

In the Cartesian coordinate system, the three-dimensional unsteady N-S equations in a conservative differential form on a moving mesh can be written as

$$
\frac{\partial}{\partial t} \int_{\Omega} \vec{W} d \Omega+\oint_{\partial \Omega}\left(\vec{F}_{c}-\vec{F}_{v}\right) d S=0
$$

Where $t$ is time, $\Omega$ is the control volume and $\partial \Omega$ is the volume surface, $\vec{W}$ is the conserved variables, $\vec{F}_{c}$ and $\vec{F}_{v}$ are convective part and viscosity part. The finite element method is used to solve the equations with $k-\omega$ turbulence model. A second order cell-centered scheme is use.

The elevator operates at $12 \mathrm{~m} / \mathrm{s}$, thus a $12 \mathrm{~m} / \mathrm{s}$ velocity inlet is used in the entrance of the elevator well. The pressure outlet boundary condition is used at the exit. The elevator car is station wall, while the elevator well is moving wall with the speed of $12 \mathrm{~m} / \mathrm{s}$. Symmetric boundary conditions are used at the symmetric planes.

\section{Results and discussion}

The drags of the high speed elevator systems are shown in Fig 3. It is shown that the drag of elevator with no cover is as much as $1892 \mathrm{~N}$, which is the largest. With the height of elevator increases, the drags of the elevator decrease. When the height of the elevator is above $0.4 \mathrm{~m}$, a linear relationship between drags and the height is assumed. 


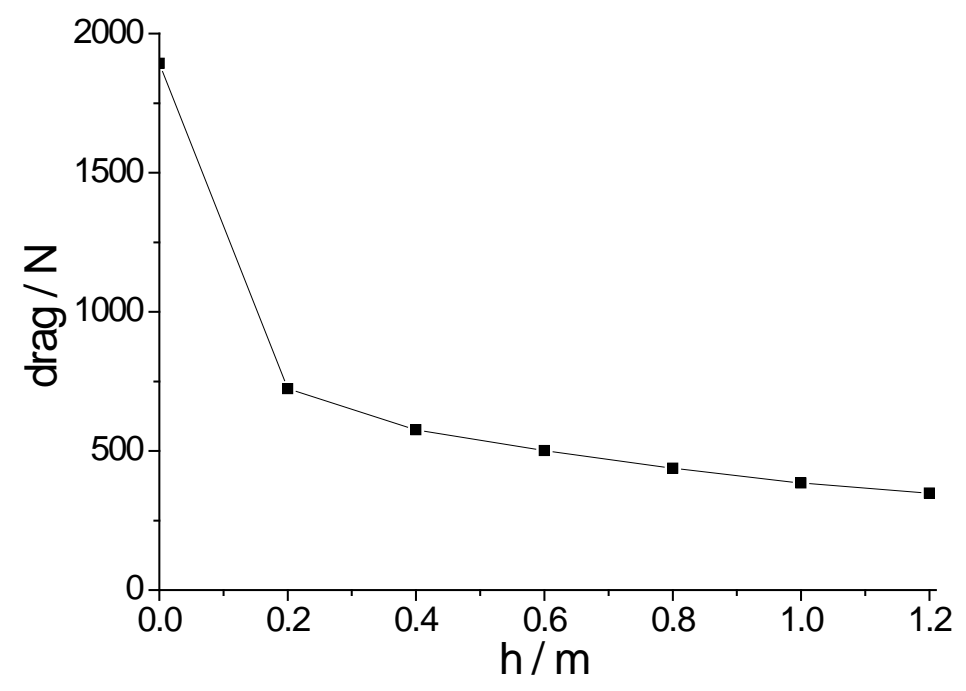

Fig.3. Drags of the elevator cars

The pressures contours of the high speed elevator car are shown in Fig 4. It is shown that the pressure of elevator with no cover is as much as $300 \mathrm{~Pa}$ and the high pressure region is quite large. With the height of elevator increases, both the drags and the high pressure region of the elevator decrease. The pressure has a homogeneous distribution at the side of the elevator box with height of $1.2 \mathrm{~m}$.

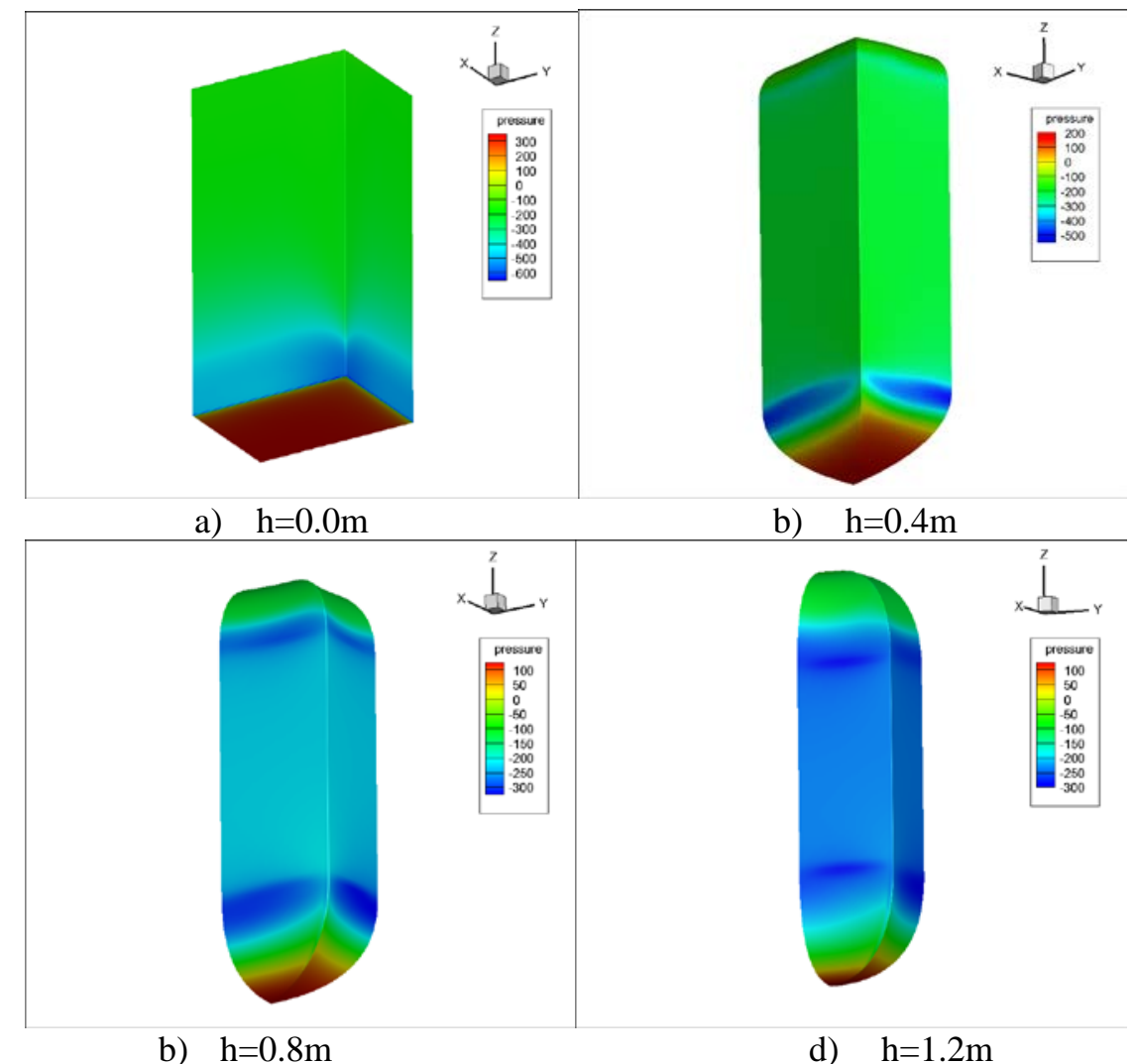

Fig.4. Pressures of the elevator car

The velocity contours of the high speed elevator car are shown in Fig 5. It is shown that the flow region of elevator with no cover has the largest velocity. With the height of elevator increases, the velocity magnitude of the elevator decrease. When the height of elevator is above $0.8 \mathrm{~m}$, the velocity contours of the flow between elevator car and the elevator well has a homogeneous distributions. 




Fig. 5. velocity contours of the high speed elevator car

\section{Conclusion}

The flows of the high speed elevator are simulated using the CFD method and the influences of the height of diversion cover on the aerodynamic performances are studied in this paper. With the height of elevator increases, the drags and pressures of the elevator decrease, the velocities of the flow region decrease. When the height of the elevator is above $0.8 \mathrm{~m}$, both the velocity and the pressure in the flow region between the elevator and the well have homogeneous distributions.

\section{Acknowledgment}

This project is supported by the Major Project of Zhejiang Bureau of Quality and Technical Supervision (NO. 20130128 ).

\section{References}

[1]. X. Zhaoa, J.M. Ding, H.H. Suna. Structural Design of Shanghai Tower for Wind Loads[J]. Procedia Engineering, 14 (2011) 1759-1767.

[2] DUAN Ying, SHEN Guo-xing. Aerodynamic testing simulation facility for high speed elevator[J]. Journal of Beijing University of Aeronautics and Astronautics, 2004, 30(5): 444-447.

[3] YANG Xiao-feng. Numerical investigation and optimization of aerodynamic characteristics of super-high speed elevators[D]. Master Thesis, Beijing: University of Aeronautics and Astronautics, 2001(in Chinese).

[4] KOBAYASHL Katsutoshi, SHIMIZU Hayato and KALHO Masayuki. Numerical simulation of unsteady turbulent flow by voxel method[J]. JSME International Journal, Ser. B, 2004, 47(3): 477-482.

[5] SHI Li-qun, LIU Ying-zheng, JIN Si-yu, CAO Zhao-min.Numerical Simulation of Unsteady Turbulent Flow Induced by Two-Dimensional Elevator Car and Counter Weight System[J]. Journal of Hydrodynamics, Ser.B, 2007,19(6):720-725. 
[6] Yang H.W, So A.T.P. A 2-Dimensional Aerodynamic Model for Super high speed Elevators. International Journal of Elevator Engineering. 1998, (2): 19 32

[7] W.D.Zhu, L.J.Teppo. Design and Analysis of a Scaled Model of a High-rise High-speed Elevator. Journal of Sound and Vibration. 2003, 26(4): 707 731 